\title{
Evidence-based analysis of self-expanding metallic stent as a bridge to surgery versus emergency surgery for colon cancer
}

\author{
“...the best strategy to evaluate the long-term impact of self-expanding \\ metallic stents is via a multicenter randomized controlled trial. However, \\ such trial would require $>500$ patients per group."
}

\section{Alberto Mangano ${ }^{*, 1} \&$ Roger W Motson ${ }^{1,2}$}

First draft submitted: 2 December 2015; Accepted for publication: 22 April 2016; Published online: 17 May 2016

In the UK, bowel cancer is the fourth most common cancer [1]. Bowel cancer incidence rates have increased by $6 \%$ over the last decade [1], approximately 1.36 million new cases worldwide in 2012 [1]. The 5 -year survival rate is $90 \%$ for localized stage; $70 \%$ for regional and $10 \%$ for distant metastatic cancer [2-4].

Up to $30 \%$ of colorectal carcinomas may present with an acute obstruction leading to potential colonic necrosis, perforation, bacterial translocation, sepsis and electrolyte/fluid imbalance. Hence, decompression of the obstructed colon, either by stenting or by surgical intervention is advisable. Left-sided obstruction is more common than right-sided obstruction [5].

5-year survival rates for colorectal carcinoma presenting as an emergency are worse and metastatic disease/local spread is more common than for elective resections $[5,6]$. For acute right-sided colonic obstruction, there is consensus that one-stage surgical treatment by primary resection and anastomosis is the treatment of choice [5,7]. There continues to be scientific debate about the best possible surgical strategy for left-sided obstruction.
De facto, colonic resection in an emergency setting carries an increased morbidity $(\sim 50 \%)[5-6,8]$ and mortality $(\sim 15 \%$ for emergency surgery vs $6 \%$ for elective surgery) [5]. In this context, the use of a self-expanding metallic stent (SEMS) as a bridge to surgery is a possible alternative to emergency surgery. However, there are some oncological concerns in terms of outcome.

\section{Evidence-based analysis}

Tan et al. [5] published a meta-analysis of SEMS in left-colonic cancer in which four randomized controlled trials (RCTs) [9-12] were included. In-hospital mortality was $5.9 \%$ in the emergency surgery group versus $6.95 \%$ in the SEMS group, though this difference was not statistically significant [5]. Furthermore, primary anastomosis rates were increased by SEMS (risk ratio [RR]: 1.58;95\% CI: 1.22-2.04; $\mathrm{p}<0.001)$ [5]. Additionally, clinically evident perforation occurred in almost $7 \%$ of the cases in SEMS arm and there was a silent perforation (detected on histological examination) in almost $15 \%$ of cases [11,12]. There was no significant difference in stoma rates

\section{KEYWORDS}

- colon cancer $\bullet$ guidelines

- meta-analysis • rectal cancer

- self-expanding metallic stent

“The 5-year survival rate is 90\% for localized stage; 70\% for regional and $10 \%$ for distant metastatic cancer." 


\author{
"Self-expanding metallic \\ stent has been considered \\ to be a promising strategy \\ in emergency surgery since \\ it theoretically combines \\ the advantages of \\ two-stage surgery ... with \\ one-stage surgery..."
}

between the two approaches [5], nor was there a significant difference for anastomotic leak [11]. The 30-day reoperation rates were $12.9 \%$ for SEMS versus $22.9 \%$ for emergency surgery [5].

Another relevant meta-analysis was published by Huang et al. [13] with five randomized controlled trials analyzed [9-12,14]. These authors found that elective surgery after SEMS may potentially improve the patients' quality of life and postoperative recovery. The SEMS group achieved significantly better rates of permanent stoma, primary anastomosis, wound infection and overall complications. Notably, there was no significant difference in anastomotic leakage/ mortality/intra-abdominal infection. An important point presented by Huang et al. [13] is that the benefit of SEMS may only be achieved in high-volume centers.

Furthermore, Huang et al. [13] assessed the effect of stent application on long-term prognosis: there was no statistical significance for overall survival rate, disease-free interval and recurrence rate. They were unable to assess the oncological impact of SEMS because survival and recurrence were not end points in the randomized trials analyzed [9-12,14]. The authors concluded that SEMS may be a safe and effective bridge to subsequent surgery in patients with obstructing left-sided colon cancer. SEMS can significantly improve one-stage surgery rates, decrease the rate of permanent stoma and reduce wound infection. Sabbagh et al. [15], in their retrospective study, showed a lower 5-year recurrence-free rate and a lower 5-year survival rate in the SEMS group. In the meta-analysis by Zhang et al. there was no significant difference in long-term survival rate, similar to Huang's findings $[13,16]$.

Moreover, they found a significantly higher primary anastomosis rate and lower complication rate for the SEMS bridge to surgery group versus the emergency surgery group. There was no significant difference in the 30-day postoperative mortality or permanent colostomy rate. In this meta-analysis and in the study by de Ceglie et al. [17] more retrospective papers were included; there was strong heterogeneity among the considered papers and the SEMS procedure had advantages over emergency surgery (reduced infection, reduction of stoma creation, increase in primary anastomosis, successful primary anastomosis and fewer other morbidities). The approaches were similar regarding long-term survival, preoperative mortality and length of hospital stay. However, some potential bias may be present in these two meta-analyses, such as sample heterogeneity and too many retrospective papers considered in their analyses.

Ye et al. [18] in another meta-analysis concluded that SEMS is not obviously more advantageous than emergency surgery. Another review and meta-analysis have been performed by Cirocchi et al. [19]. The clinical success rate was significantly higher in the emergency surgery group. There was no difference in the overall complication rate or in the 30-day postoperative mortality. The overall survival was not analyzed. However, in this study SEMS, as a bridge to surgery, had some advantages: the primary anastomosis rate was significantly higher in the stent group versus the emergency surgery group (64.9 vs 55\%; $\mathrm{p}=0.003$ ); the overall stoma rate was significantly lower in the stent group compared with the emergency surgery group (45.3 vs $62 \% ; p=0.02)$. There were no significant differences between the two groups in terms of permanent stoma rate, anastomotic leakage rate and intra-abdominal abscess rate.

In the Cennamo et al. meta-analysis [20], the authors concluded that SEMS placement improved primary anastomosis, stoma formation and permanent stoma rates, but there was no improvement in either morbidity or mortality [20]. Given the modest/heterogeneous sample size of the included studies, further RCTs are necessary enrolling a larger number of patients, assessing long-term results (standard quality of life/overall survival) and cost-effectiveness analysis [5,19].

\section{Discussion}

SEMS has been considered to be a promising strategy in emergency surgery since it theoretically combines the advantages of two-stage surgery (preparation for elective surgery correcting any patient's acute clinical derangements and at the same time treating the obstruction) with one-stage surgery (a single surgical procedure and no stoma). Moreover, SEMS could prevent some potential risks associated with the surgical approach (mortality, morbidity, length of hospital stay and economic impact) [15]. However, the precise role of SEMS as a bridge to surgery is still a matter of debate since there are heterogenous and conflicting data (even among the aforementioned meta-analyses) with regard to complications and outcome. Moreover, when this approach has been tested via RCTs, the majority of trials have been stopped prematurely for safety concerns (high morbidity on the SEMS arm) [11-12,15]. The 
overall clinical/technical success rate had a wide range of variability among authors from 30 to $92 \%$ [11-12,21-22]. Of note, SEMS placement is an operator-dependent procedure and complication rate is decreased when performed by an expert clinician [21]. In fact, expertise could be relevant also in terms of outcome (i.e., a possible perforation related to poor/limited expertise in SEMS could potentially influence the outcome). Small et al. [21] state that the degree of occlusion by the tumor is extremely important in SEMS complication rate. This being a very tight stricture potentially creates difficulties for stent placement and a completely occluded lumen is often friable and prone to microperforations.

Furthermore, silent perforation could increase neoplastic dissemination and seeding. In particular, Kim et al. [23] showed a decreased 5-year overall and disease-free survival rates in patients that underwent SEMS as a bridge to surgery for left colonic tumors, but the inferior outcome could have been linked to more advanced disease presenting in emergency bowel obstruction [6].

On the other hand, in emergency left colonic obstructions, given the high risk of anastomotic leak [24], resection and colostomy is the most common surgical procedure [25,26]. A subsequent reversal procedure with anastomosis can be performed in up to $60 \%$ of cases but there is a considerable morbidity associated with reversal (up to 40\%) [5]. For these reasons, SEMS would seem to be a good therapeutic strategy in order to perform a colonic decompression, stabilization of the patient and may allow elective surgery in a safer context. However, taking into consideration the possible technical/clinical failure and the potential complications, and the conflicting results available, more robust data on the topic are needed to draw an evidence-based conclusion.

Moreover, SEMS costs more than the emergency surgery [5]. The only authors who showed no differences in terms of costs were Alcantara et al. [9].

The main debate at present is the long-term oncological outcome of SEMS. There remains concern that stent insertion may increase the risk for dissemination-related metastasis [27] although other authors have found that there are not any detectable differences in terms of outcome [12,23].

Sabbagh et al. [15] challenged the safety of SEMS showing that the 5-year survival rate and 5-year tumor-free survival rate in SEMS group were significantly lower than those in the emergency surgery group (even after excluding cases with perforation and metastasis), although semielective surgery after SEMS achieved higher lymph node harvest and a higher postoperative chemotherapy rate.

In conclusion, there are plenty of conflicting/ not robust results shown by different authors. When a new technique or technology is introduced, there needs to be an evidence-based validation achieving technical and procedural standardization, which is mandatory in order to maintain the obtained standards, for safety and for repeatability and to compare results via an evidence-based rigorous methodology [28]. In particular, the best strategy to evaluate the long-term impact of SEMS is via a multicenter randomized controlled trial. However, such trial would require $>500$ patients per group [9]. Hence, at the moment there are no trials on this topic with a primary endpoint of long-term survival. For these reasons, SEMS is not at this stage a procedure that has been standardized and its results have not been properly studied/compared in terms of oncological outcome when used as a bridge to surgery. There is a need for more studies, in particular RCTs with sufficient power to better investigate the impact of SEMS in terms of outcome and better clarify the areas of current concern.

\section{Financial \& competing interests disclosure}

The authors have no relevant affiliations or financial involvement with any organization or entity with a financial interest in or financial conflict with the subject matter or materials discussed in the manuscript. This includes employment, consultancies, honoraria, stock ownership or options, expert testimony, grants or patents received or pending, or royalties.

No writing assistance was utilized in the production of this manuscript.

\section{References}

1 Cancer Research UK. www.cancerresearchuk.org

2 Siegel R, Desantis C, Jemal A. Colorectal cancer statistics. CA Cancer J. Clin. 64(2), 104-117 (2014)
3 Jemal A, Clegg LX, Ward E et al. Annual report to the nation on the status of cancer, 1975-2001, with a special feature regarding survival. Cancer 101(1), 3-27 (2004).

4 Ries LAG, Melbert D, Krapcho M et al. SEER Cancer Statistics Review, 1975-2005.
Bethesda, MD, USA (2008). http://seer.cancer.gov/archive/csr/1975_2005

5 Tan CJ, Dasari BVM, Gardiner K. Systematic review and meta-analysis of randomized clinical trials of self-expanding metallic stent as a bridge to surgery versus emergency 
surgery for malignant left-sided large bowel obstruction. Br. J. Surg. 99, 469-476 (2012).

6 McArdle CS, Hole DJ. Emergency presentation of colorectal cancer is associated with poor 5-year survival. Br. J. Surg. 91(5), 605-609 (2004).

7 Finan PJ, Campbell S, Verma R et al. The management of malignant large bowel obstruction: ACPGBI position statement. Colorectal Dis. 9(Suppl. 4), 1-17 (2007).

8 Iversen LH, Bülow S, Christensen IJ et al. Danish Colorectal Cancer Group. Postoperative medical complications are the main cause of early death after emergency surgery for colonic cancer. Br. J. Surg. 95(8), 1012-1019 (2008).

9 Alcántara M, Serra-Aracil X, Falcó J et al. Prospective, controlled, randomized study of intraoperative colonic lavage versus stent placement in obstructive left-sided colonic cancer. World J. Surg. 35(8), 1904-(2011).

10 Cheung HY, Chung CC, Tsang WW et al. Endolaparoscopic approach vs conventional open surgery in the treatment of obstructing left-sided colon cancer: a randomized controlled trial. Arch. Surg. 144(12), 1127-1132 (2009).

11 Pirlet IA, Slim K, Kwiatkowski F et al. Emergency preoperative stenting versus surgery for acute left-sided malignant colonic obstruction: a multicenter randomized controlled trial. Surg. Endosc. 25(6), 1814-1821 (2011).

12 van Hooft JE, Bemelman WA, Oldenburg B et al. collaborative Dutch Stent-In study group. Colonic stenting versus emergency surgery for acute left-sided malignant colonic obstruction: a multicentre randomised trial. Lancet Oncol. 12(4), 344-352 (2011).

13 Huang X, Lv B, Zhang S, Meng L. Preoperative colonic stents versus emergency surgery for acute left-sided malignant colonic obstruction: a meta-analysis. J. Gastrointest. Surg. 18(3), 584-591 (2014).

14 Ho K-S, Quah H-M, Lim J-F et al. Endoscopic stenting and elective surgery versus emergency surgery for left-sided malignant colonic obstruction: a prospective randomized trial. Int. J. Colorectal Dis. 27(3), 355-362 (2012).

15 Sabbagh C, Browet F, Diouf M et al. Is stenting as "a bridge to surgery" an oncologically safe strategy for the management of acute, left-sided, malignant, colonic obstruction? A comparative study with a propensity score analysis. Ann. Surg. 258(1), 107-115 (2013).

16 Zhang Y., Shi J., Shi B. et al. Self-expanding metallic stent as a bridge to surgery versus emergency surgery for obstructive colorectal cancer: a metaanalysis. Surg. Endosc. 26(1), 110-119 (2012).

17 de Ceglie A., Filiberti R., Baron T. H. et al. A meta-analysis of endoscopic stenting as bridge to surgery versus emergency surgery for left-sided colorectal cancer obstruction. Crit. Rev. Oncol. Hematol. 88(2), 387-403 (2013).

18 Ye GY, Cui Z, Chen L et al. Colonic stenting vs emergent surgery for acute left sided malignant colonic obstruction: a systematic review and meta-analysis. World J. Gastroenterol. 18(39), 5608-5615 (2012).

19 Cirocchi R, Farinella E, Trastulli S et al. Safety and efficacy of endoscopic colonic stenting as a bridge to surgery in the management of intestinal obstruction due to left colon and rectal cancer: a systematic review and meta-analysis. Surg. Oncol. 22(1), 14-21 (2013).

20 Cennamo V, Luigiano C, Coccolini F. Meta-analysis of randomized trials comparing endoscopic stenting and surgical decompression for colorectal cancer obstruction. Int. J. Colorectal Dis. 28(6), 855-863 (2013).

21 Small AJ, Coelho-Prabhu N et al. Endoscopic placement of self-214 expandable metal stents for malignant colonic obstruction: long-term outcomes and complication factors.

Gastrointest. Endosc. 71(3), 560-572 (2010).

22 Sebastian S, Johnston S, Geoghegan T et al. Pooled analysis of the efficacy and safety of self-expanding metal stenting in malignant colorectal obstruction. Am. J. Gastroenterol. 99(10), 2051-2057 (2004).

23 Kim JS, Hur H, Min BS et al. Oncologic outcomes of self-expanding metallic stent insertion as a bridge to surgery in the management of left-sided colon cancer obstruction: comparison with nonobstructing elective surgery. World J. Surg. 33(6), 1281-1286 (2009).

24 Kingham TP, Pachter HL. Colonic anastomotic leak: risk factors, diagnosis, and treatment. J. Am. Coll. Surg. 208(2), 269-278 (2009).

25 Breitenstein S, Rickenbacher A, Berdajs D et al. Systematic evaluation of surgical strategies for acute malignant left-sided colonic obstruction. Br. J. Surg. 94(12), 1451-1228 (2007).

26 Morris EJ, Taylor EF, Thomas JD et al. Thirty-day postoperative mortality after colorectal cancer surgery in England. Gut 60 (6), 806-813 (2011).

27 Maruthachalam K, Lash GE, Shenton BK et al. Tumour cell dissemination following endoscopic stent insertion. Br. J. Surg. 94(9), 1151-1154 (2007).

28 Mangano A., Mangano A., Lianos GD et al. Circulating free DNA in plasma or serum as biomarkers of carcinogenesis in colon cancer Future Oncol. 11(10), 1455-1458 (2015). 